Supplemental Information

\title{
BPEI-Induced Delocalization of PBP4 Potentiates $\beta$-lactams Against MRSA
}

Melissa A. Hill', Anh K. Lam ${ }^{1}$, Patricia Reed ${ }^{2}$, Madeline C. Harney ${ }^{1}$, Beatrice A. Wilson ${ }^{1}$, Erika

L. Moen ${ }^{1}$, Summer N. Wright ${ }^{1}$, Mariana G. Pinho ${ }^{2}$, Charles V. Rice ${ }^{1 *}$ 

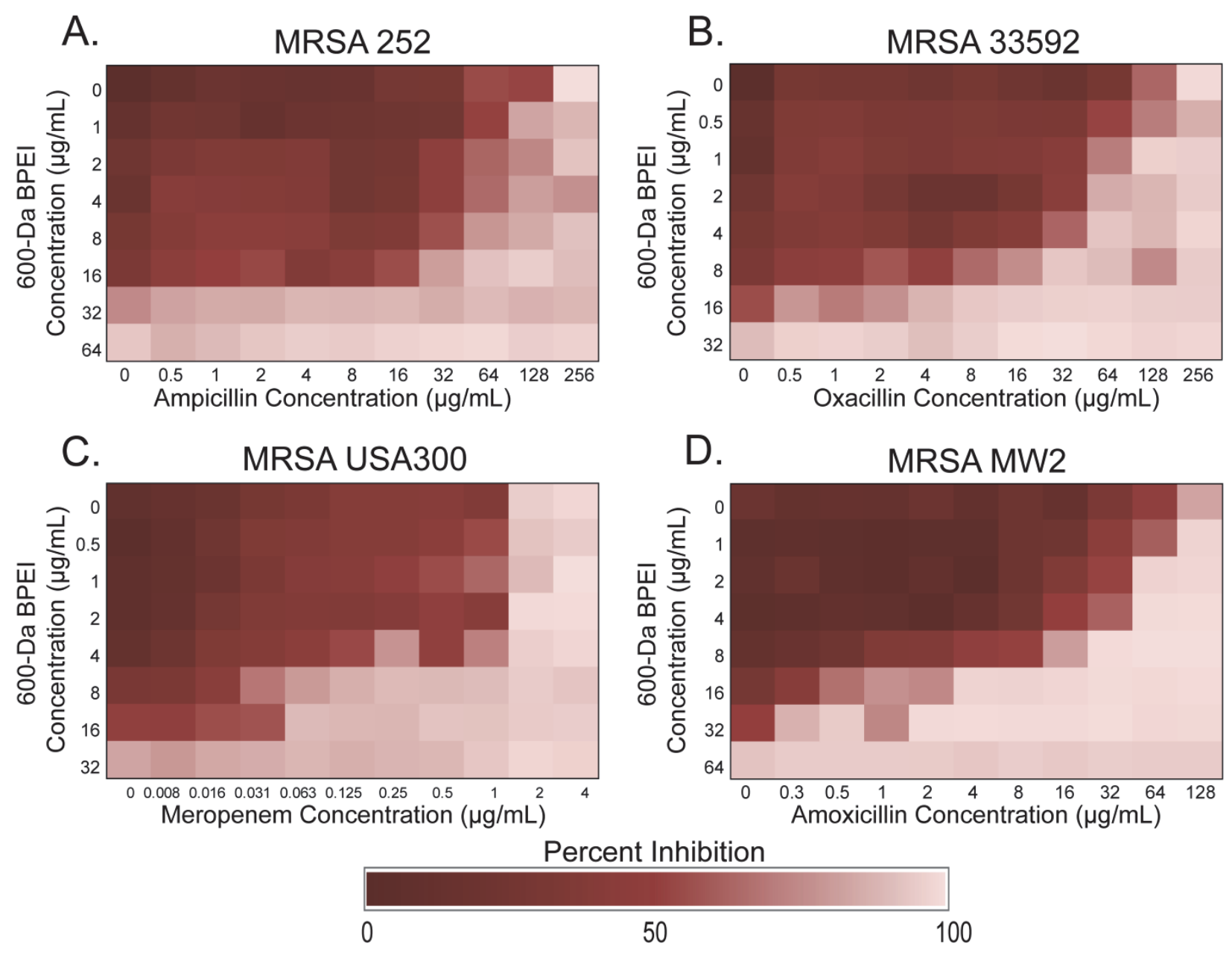

Figure S1. Checkerboard assays show synergy between BPEI and $\beta$-lactams on MRSA 252, MRSA 33592, MRSA USA300 and MRSA MW2. BPEI had synergy with ampicillin against hospital-acquired MRSA 252 (A). BPEI had synergy with oxacillin against MRSA 33592 (B), with meropenem against MRSA USA300 (C), and with amoxicillin agisnt MRSA MW2 (D). Each assay was performed as three separate trials and the presented data is shown as the average change in OD 600 . 

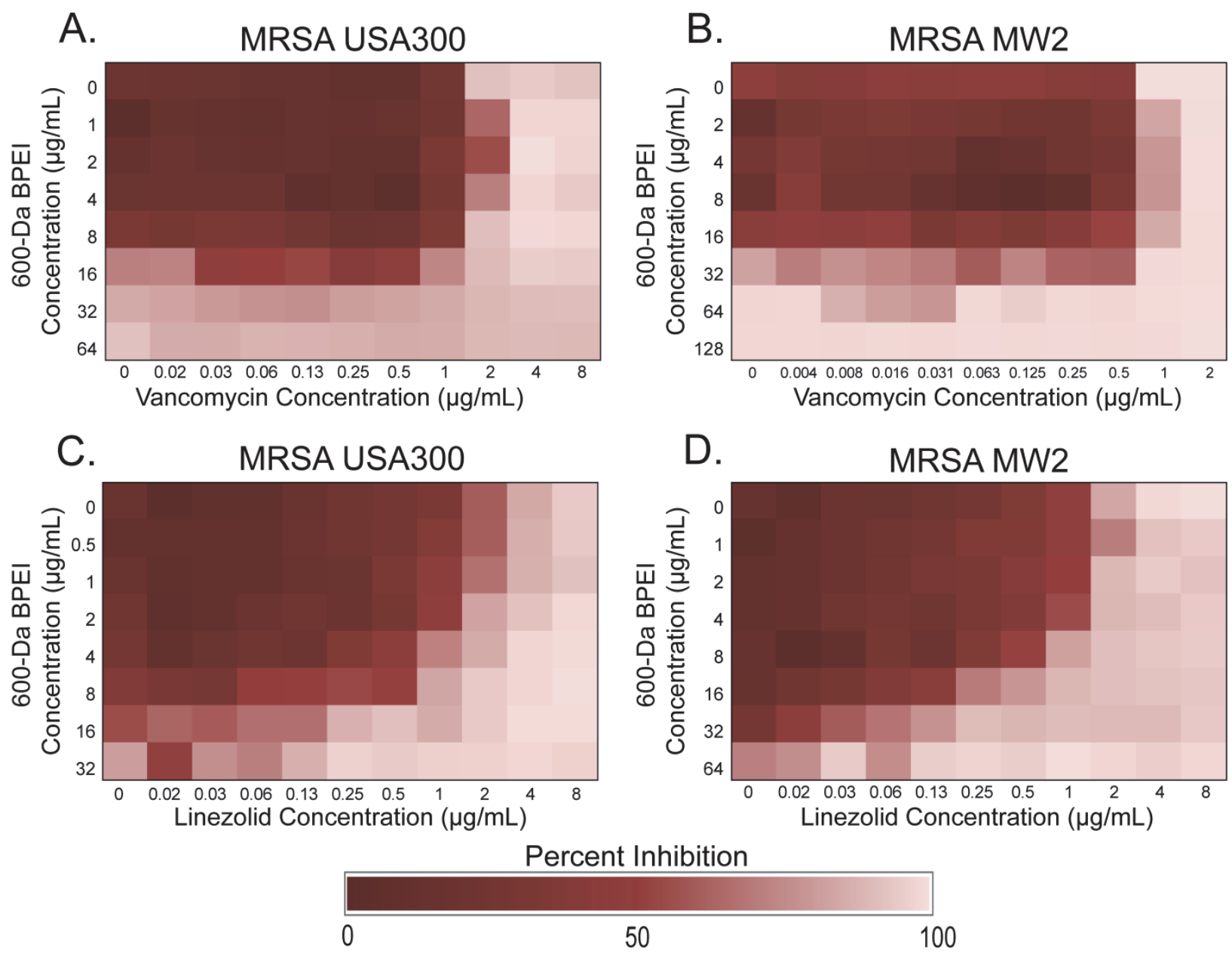

Figure S2. BPEI does not potentiate synergy with vancomycin or linezolid. Checkerboard assays show that BPEI did not have synergy with vancomycin $(A, B)$ or linezolid $(C, D)$ against MRSA USA300 or MRSA MW2. Each assay was performed as three separate trials and the presented data is shown as the average change in OD 600 . 


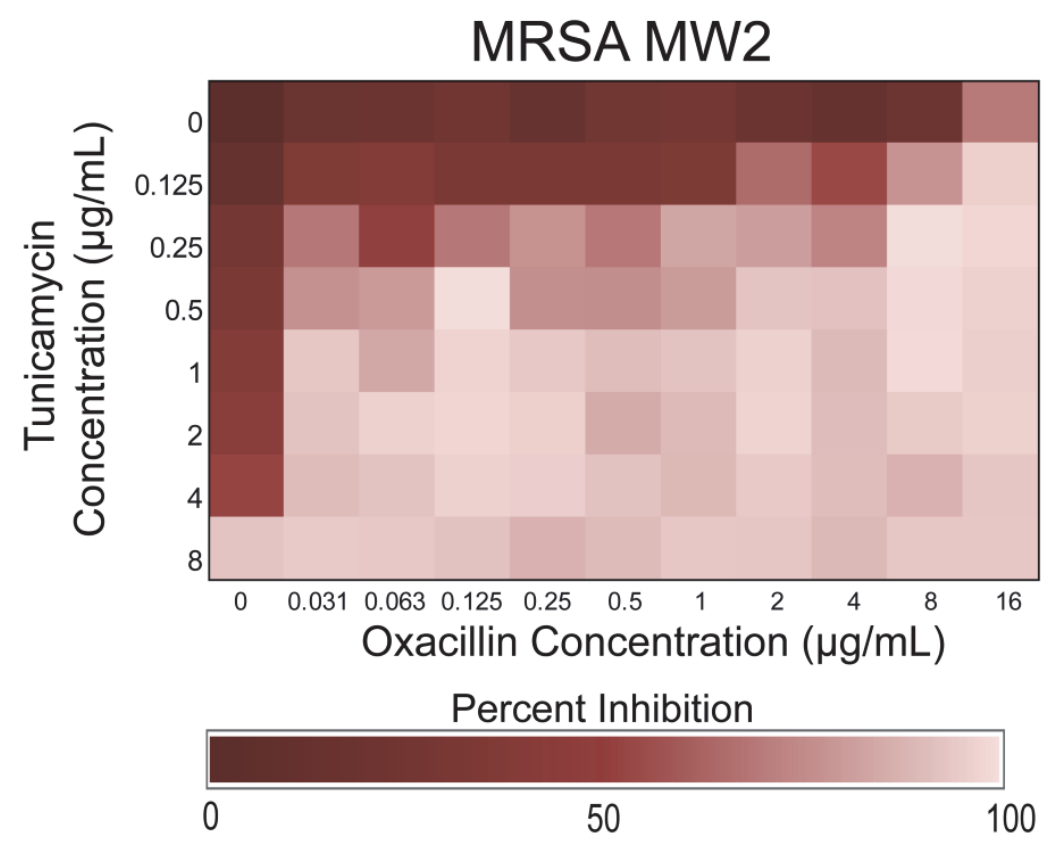

Figure S3. Tunicamycin potentiates oxacillin against MRSA MW2. The data is the average of three separate trials and reported as the average change in OD600. 
A-B. MRSA COL PBP2:GFP, No BPEI, 60 minutes
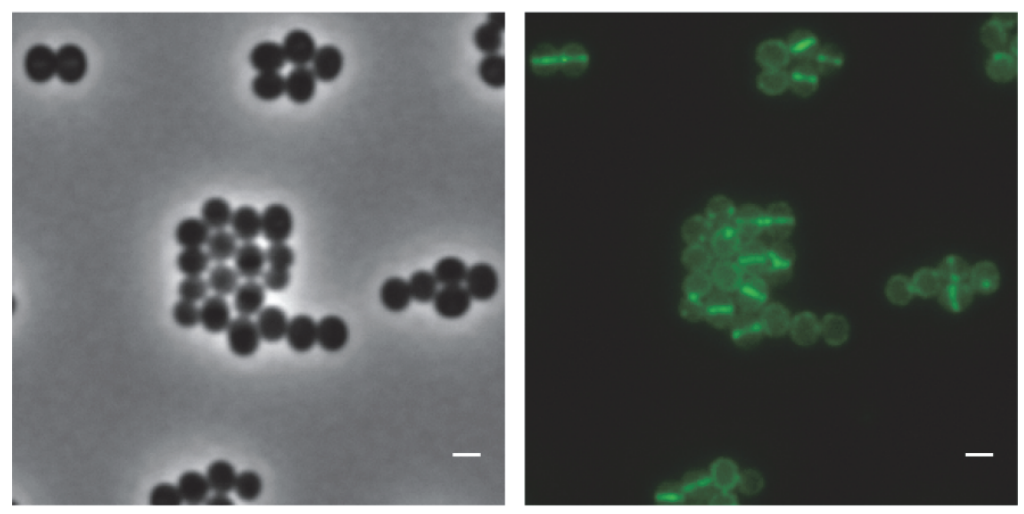

C-D. MRSA COL PBP2:GFP, $64 \mu \mathrm{g} / \mathrm{mL}$, BPEI 60 minutes
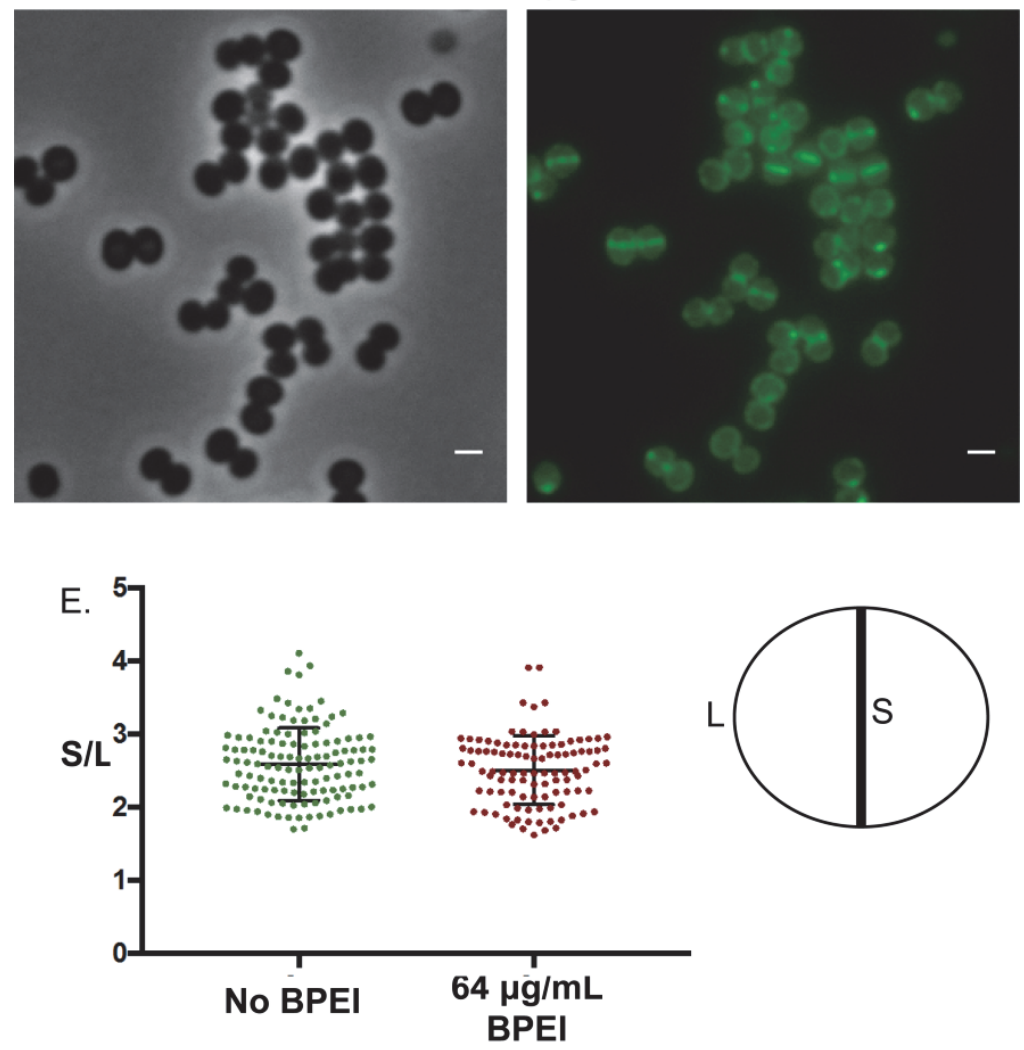

Figure S4. Fluorescence images of MRSA Col PBP2:GFP shows that PBP2:GFP primarily localizes at the division septum of dividing cells (A, B). BPEI does not significantly alter localization patterns of PBP2:GFP (C, D). Quantitative analysis of the fluorescence intensity at the division septum versus fluorescence intensity at non-septum regions was performed for untreated and BPEI-treated samples. Analysis was done for 100 cells of each sample that show a visible closed septum. The PBP2 for untreated cells $(\mathrm{S} / \mathrm{L}=2.59 \pm 0.04)$ localized similar to those in the BPEI-treated samples $(\mathrm{S} / \mathrm{L}=2.50 \pm 0.05)$. Scale bar $=1 \mu \mathrm{m}$. 
A-B. MRSA COL FtsZ:CFP, No BPEI, 60 minutes
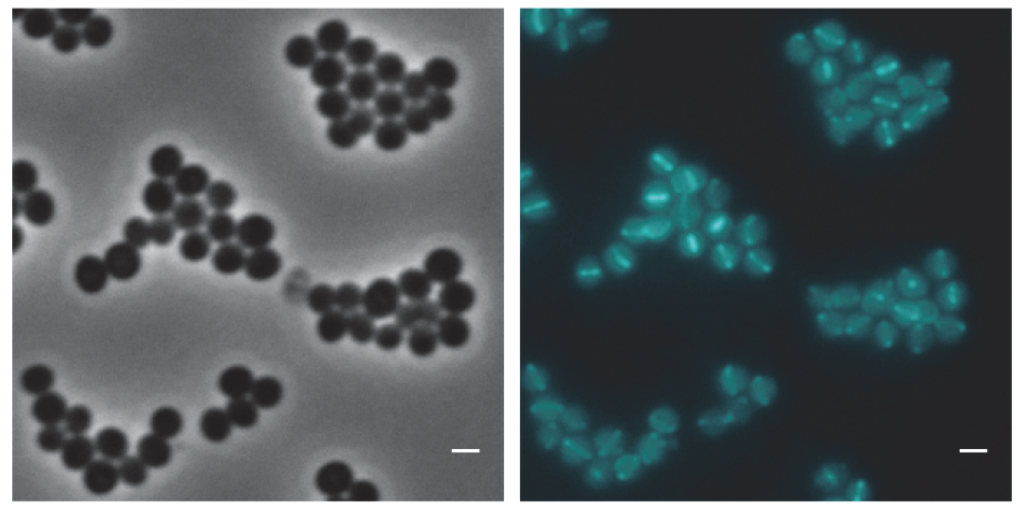

C-D. MRSA COL FtsZ:CFP, $64 \mu \mathrm{g} / \mathrm{mL}$, BPEI 60 minutes
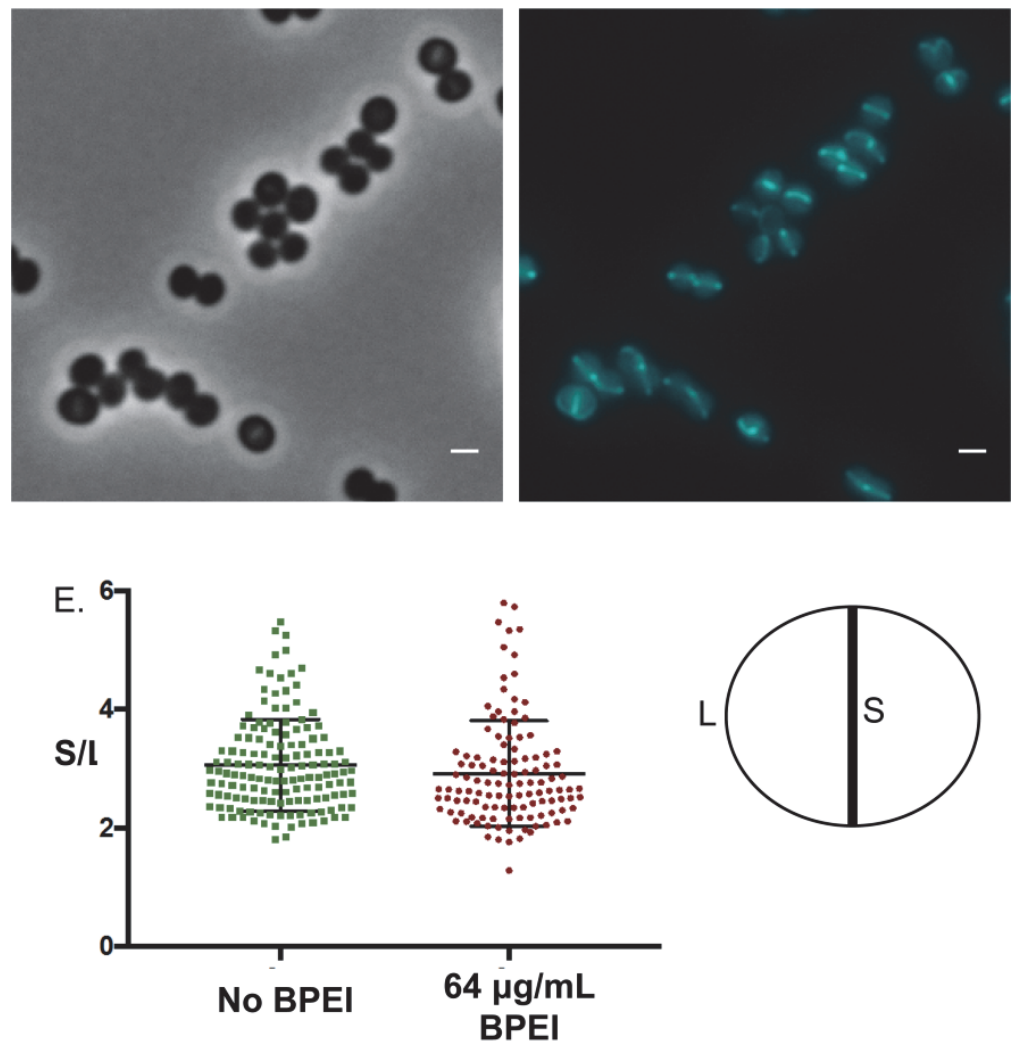

Figure S5. Fluorescence images of MRSA Col FtsZ:CFP shows that FtsZ primarily localizes at the division septum of dividing cells (A, B). BPEI does not significantly alter localization patterns of FtsZ (C, D). Quantitative analysis of the fluorescence intensity at the division septum versus fluorescence intensity at non-septum regions was performed for untreated and BPEItreated samples. Analysis was done for 100 cells of each sample that show a visible closed septum. The FtsZ for untreated cells $(\mathrm{S} / \mathrm{L}=2.91 \pm 0.08)$ localized similar to those in the BPEItreated samples $(\mathrm{S} / \mathrm{L}=3.06 \pm 0.06)$. Scale bar $=1 \mu \mathrm{m}$. 


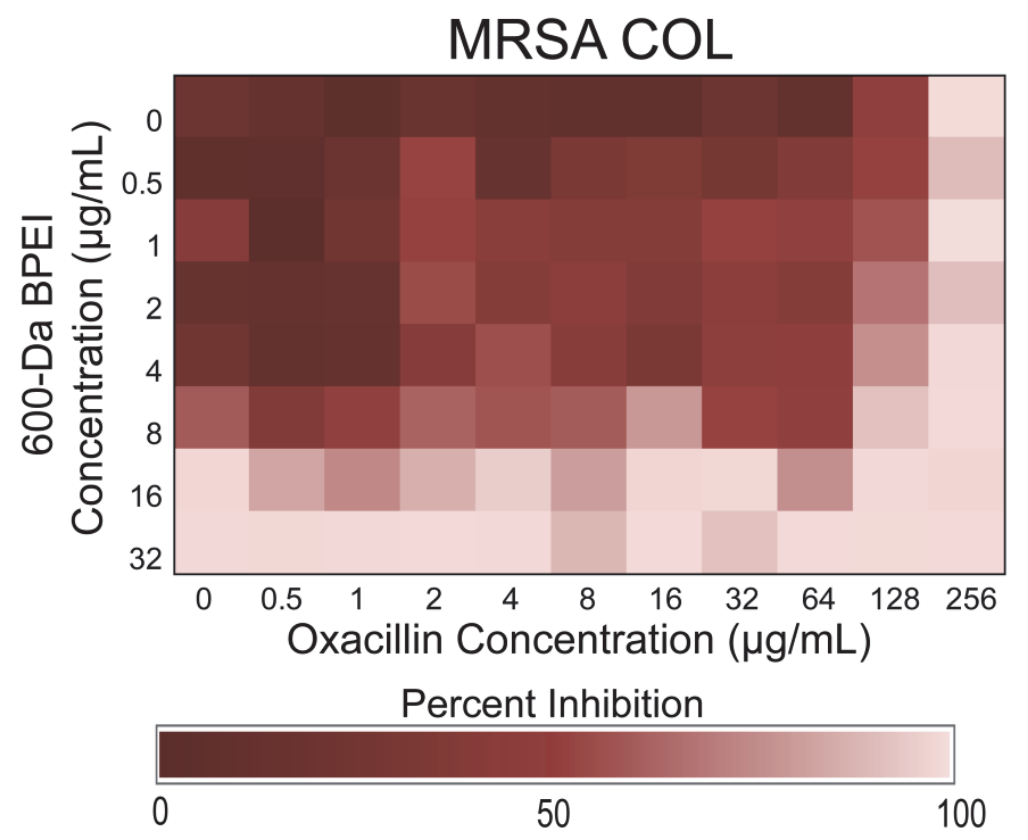

Figure S6. BPEI is unable to potentiate oxacillin against MRSA COL. The data is the average of three separate trials and reported as the average change in $\mathrm{OD}_{600}$. 
Table S1. BPEI prevents MRSA USA300 cells from separating efficiently.

\begin{tabular}{|c|cc|}
\hline & Untreated (\%) & Treated (\%) \\
\hline No Visible Septa & $75 \%$ & $53 \%$ \\
Complete Septa & $12 \%$ & $24 \%$ \\
Incomplete Septum & $13 \%$ & $23 \%$ \\
\hline
\end{tabular}

Notes: 200 cells from each sample were analyzed from TEM images to determine the level of septal formation. 